\title{
UMA AVALIAÇÃO DO NÍVEL DE EVIDENCIAÇÃO DAS COMPANHIAS ABERTAS, NO BRASIL, NO TOCANTE AOS INSTRUMENTOS FINANCEIROS
}

\author{
Jorge Vieira da Costa Junior \\ Mestre em Ciências Contábeis pela UERJ \\ E-mail: jvieiracosta@ig.com.br
}

\section{RESUMO}

O propósito deste trabalho é o de levantar o quadro atual da política de evidenciação de instrumentos financeiros por parte das companhias abertas no Brasil, através de suas demonstrações contábeis (data base de 31.12.2000).

Uma pesquisa junto a 215 companhias abertas foi levada a efeito visando a avaliar se a divulgação de fatores de risco de mercado e de sua gestão foi feita adequadamente (de forma qualitativa e quantitativa), se as estratégias dispensadas ao uso dos instrumentos financeiros foram adequadamente reveladas, se foram computados e revelados os valores justos dos instrumentos financeiros, enfim, se os ditames da Instrução CVM no 235/95, de 23.03.95, foram observados rigorosamente pelas companhias abertas.

O resultado da pesquisa indicou que as companhias abertas no Brasil, não prestam informações adequadas em suas demonstrações contábeis, no que concerne aos instrumentos financeiros.

Palavras-chave: instrumentos financeiros, derivativos, evidenciação de riscos de mercado, companhias abertas.

\section{ABSTRACT}

This paper aimed to investigate the current stage of the disclosure policy of publicly-held companies in Brazil with respect to financial instruments, by means of the analysis of their financial statements (fiscal year ending at December $31^{\text {st }}$ 2000).

A research of 215 publicly-held companies was realized in order to evaluate the adequate disclosure (qualitatively and quantitatively speaking) of risk market factors and their management, the adequate revelation of the strategies for using financial instruments, the adequate calculation and revelation of the fair values of the financial instruments, and finally, if the paragraphs of CVM Instruction no. 235/ 95, from March $23^{\text {rd }} 1995$, were strictly observed by the publicly-held companies. Moreover, the role of the independent auditors in this context was evaluated through the analysis of their audit opinions.

The research result indicated that the publicly-held companies in Brazil do not render appropriate information about the financial instruments in their financial statements, with the consent of their independent auditors. Part of the responsibility may also be attributed to the Regulator, due to the absence of a more educational and explanatory regulation (of the "how to do" kind).

Keywords: financial instruments, derivatives, market risk disclosure, publicly-held companies. 


\section{INTRODUÇÃO}

\subsection{Apresentação do problema}

Em 1995, a Comissão de Valores Mobiliários CVM, por intermédio da Instrução CVM no 235/ 95, de 23.03.1995, disciplinou pela primeira vez o tratamento contábil a ser dado aos Instrumentos Financeiros pelas companhias abertas. A dita norma concentrou-se exclusivamente na questão da evidenciação (disclosure) ${ }^{1}$.

Não foi por acaso que a autarquia regulou oportunamente a matéria. No início da década de 90 , vale dizer, os instrumentos financeiros ganharam notoriedade no âmbito internacional, por conta de colapsos financeiros que assolaram grandes instituições, como por exemplo "Orange County" (nos EUA, 1993/1994), "Metallgesellschaft Refining \& Marketing" (nos EUA, 1992/1993), "Barings Bank" (em Cingapura, 1994/1995), entre outras.

Passados 6 anos desde a emissão do citado normativo, ao longo dos quais a CVM interveio reiteradas vezes advertindo as companhias abertas pela sua inobservância ${ }^{2}$, um estudo acadêmico mais aprofundado do tema reputou-se relevante e pertinente. Esse é o propósito deste trabalho, ou seja, investigar o nível de observância da Instrução CVM no 235/95 por parte das companhias abertas.

\subsection{Caracterização do problema}

Problema Acadêmico: Qual o quadro atual das companhias abertas brasileiras, no tocante à divulgação de informações relativas a instrumentos financeiros, requerida pela CVM?

A investigação do problema tem como pressuposto o fato de as companhias abertas brasileiras não atenderem ao mínimo requerido pela Instrução CVM no 235/95, de 23.03.95, em termos de informações relacionadas aos instrumentos financeiros. Tal premissa tem por norte manifestações da CVM através de seu corpo técnico.

São construídas as seguintes hipóteses, à luz dos ensinamentos de STEVENSON (1981, p. 223):

\begin{tabular}{|l|l|}
\hline Hipótese Nula & $\begin{array}{l}\mathrm{H}_{0} \text { : as companhias abertas não prestam informações mínimas requeridas } \\
\text { pela Instrução CVM no 235/95 }\end{array}$ \\
\hline Hipótese Alternativa & $\begin{array}{l}\mathrm{H}_{1} \text { : as companhias abertas prestam informações mínimas requeridas } \\
\text { pela Instrução CVM no 235/95. }\end{array}$ \\
\hline
\end{tabular}

Há de se dizer que algumas premissas foram consideradas no curso da pesquisa. Primeiro, o fato de não haver instrumentos financeiros identificáveis nas demonstrações contábeis apreciadas, tampouco notas explicativas elaboradas, foi tomado como uma evidência da não utilização de instrumentos financeiros por uma companhia (mesmo aqueles de natureza off-balance).

Segundo, em decorrência da ausência de orientação por parte da CVM quanto à diretriz a ser observada para divulgação de riscos (se deve ser qualitativa, quantitativa ou ambas), foi considerado como requerido o disclosure qualitativo e quantitativo de risco.
Ademais, só foram enquadradas como companhias que adotam o disclosure qualitativo de risco aquelas que identificaram em nota explicativa o fator ou fatores de risco a que estão sujeitas e, quando fosse o caso, o instrumento ou instrumentos utilizado(s) para hedgeá-lo(s).

\subsection{Metodologia científica}

Dessa forma, uma amostragem do universo total de companhias abertas registradas na CVM foi levada a efeito, com o propósito de se verificar: (1) se a companhia tem instrumento financeiro; (2) se a companhia faz a divulgação qualitativa de riscos; (3) se a

\footnotetext{
${ }^{1}$ Em verdade, o artigo $5^{\circ}$ da Instrução CVM n ${ }^{\circ} 235 / 95$, único dispositivo da norma a abordar o registro contábil de instrumentos financeiros, tratou isoladamente da contabilização das então denominadas operações vinculadas, criadas pelo mercado no início do processo de privatização.

${ }^{2}$ Ofícios Circulares foram emitidos, em conjunto, pela Superintendência de Normas Contábeis e de Auditoria e pela Superintendência de Relações com Empresas da autarquia, direcionados a Diretores de Relações com Investidores e a Auditores Independentes (Ofícios CVM/SEP/SNC/n오 04/1995, 01/1996 e 02/2000)
} 
companhia faz a divulgação quantitativa de riscos; (4) de que forma a companhia atua no mercado de instrumentos financeiros (hedge, especulação, arbitragem); (5) se a companhia divulga o fair value dos seus instrumentos financeiros.

Os dados foram tabulados em planilha eletrônica EXCEL (apêndice), grupados por auditores independentes e por tipo de entidade. Foram definidos os seguintes grupos de auditores independentes: A, B, $\mathrm{C}, \mathrm{D}$ e $\mathrm{E}$ (as denominadas "big five" da auditoria mundial); $\mathrm{F}, \mathrm{G}$ e H (as três maiores firmas nacionais por carteira de clientes); e outras (as demais firmas de auditoria registradas na CVM).

As companhias foram estratificadas nos seguintes grupos: (1) instituições bancárias;(2) companhias que participam de programas de Depositary Receipts - DR's (ADR's, GDR's, BDR's etc.); (3) companhias integrantes do índice IBOVESPA (carteira teórica de maio a agosto de 2001); e (4) demais companhias.

No enquadramento das companhias por grupo, ocorreu, por vezes, uma superposição de classes. Nessas circunstâncias, o critério utilizado foi o de fazer prevalecer o tipo (1) sobre o (2) e o (3), e o tipo (2) sobre o (3).

A importância do estabelecimento de grupos distintos de companhias (tipos 1, 2, 3, e 4) tem relação direta com a técnica de amostragem utilizada. A amostragem foi em parte estratificada e em parte sistemática. Do universo total de companhias abertas que arquivaram suas demonstrações contábeis de 31.12.2000 na CVM, 215 foram selecionadas, de acordo com o seguinte critério:

- $100 \%$ das instituições bancárias;

- $100 \%$ das companhias que participam de programas de Depositary Receipts - DR's;

-100\% das companhias integrantes do índice IBOVESPA; e

- sistematicamente, demais companhias (independente do setor econômico de atuação), na quantidade necessária para se chegar a uma amostra total de 215 companhias abertas.

O grupo "demais companhias" foi objeto da amostragem sistemática, segundo listagem gerada pelo sistema SAFIAN, desenvolvido pela Bovespa. Expurgando as companhias contidas nos três outros grupos (instituições bancárias, companhias que participam de programas de $D R$ 's e companhias que integram o Índice IBOVESPA), a cada intervalo de cinco companhias listadas, uma foi selecionada (ou a imediatamente posterior, no caso de a seleção ter identificado uma companhia já integrante da amostra).

A amostra de companhias abertas selecionadas, um total de 215 , representa mais de $\mathbf{2 5} \%$ do universo de companhias abertas registradas na CVM e mais de $\mathbf{4 0 \%}$ das companhias registradas, que arquivaram suas demonstrações contábeis de 31.12.2000 na CVM, até 30.06.2001 (data base de corte).

O critério de amostragem teve por objetivo extrair um conjunto que refletisse o universo total de companhias que efetivamente transacionam instrumentos financeiros, de modo a não prejudicar inferências sobre a população como um todo. A grande preocupação foi evitar tendenciosidades ou amostras não representativas.

Instituições bancárias, por natureza, são as que preponderantemente transacionam instrumentos financeiros, para uso próprio ou de terceiros. Companhias que participam de programas de DR's, dada a maturidade requerida para atuar em mercados de capitais mais desenvolvidos, seriam potenciais usuárias de instrumentos financeiros (pelo menos para hedgear seus riscos e reduzir, com isso, seu custo de captação), o que é uma suposição. Já as companhias integrantes do índice IBOVESPA, pela sua importância para o mercado, e por serem geralmente grandes corporações, imagina-se que transacionem instrumentos financeiros corriqueiramente, o que é também uma suposição.

\section{PESQUISA DE CAMPO}

Considerando-se o efeito das superposições, as 215 companhias, as quais representam mais de $25 \%$ das companhias abertas registradas na CVM e mais de $40 \%$ das companhias registradas que arquivaram suas demonstrações contábeis de 31.12.2000 na CVM até 30.06.2001, foram assim distribuídas:

\begin{tabular}{|lrr|}
\hline BANCÁRIA & 31 & $14,40 \%$ \\
IBOVESPA & 11 & $5,10 \%$ \\
DR'S & 67 & $31,20 \%$ \\
DEMAIS & 106 & $49,30 \%$ \\
& $\underline{215}$ & $\underline{100,00 \%}$ \\
\hline
\end{tabular}


Desse total, 192 ou 89,30\% transacionam com instrumentos financeiros (isso está refletido nas demonstrações contábeis ou notas explicativas apreciadas), enquanto 23 ou $10,70 \%$ não possuem quaisquer instrumentos financeiros em carteira, quer sejam derivativos, quer sejam não derivativos.

Um total de 5 companhias ou 2,60\%, considerando o universo de companhias que transacionam instrumentos financeiros, faz a divulgação qualitativa de seus riscos nos moldes definidos na pesquisa (identificação do fator ou fatores de risco e, quando for o caso, do instrumento adotado para fins de hedge).

Para ilustrar o resultado, reproduzem-se parci- almente notas explicativas de duas companhias que se enquadraram na divulgação qualitativa de risco. A companhia 151 descreve sua estratégia para hedgear o fator de risco de mercado taxa de juros. A companhia 215 descreve a sua estratégia para hedgear o fator risco de crédito.

\section{CIA. 151}

(b) Hedge de juros (LIBOR 6 meses)

A Companhia tem contratado operações financeiras com derivativos, visando a proteger sua exposição à LIBOR de 6 meses, decorrente de suas operações de trade finance. Em 31/12/00 estas operações eram as seguintes:

\begin{tabular}{|c|c|c|c|}
\hline Tipo & $\begin{array}{r}\text { Valores das } \\
\text { operações }\end{array}$ & Último vencimento & $\begin{array}{r}\text { Ganho (perda) não } \\
\text { Realizado (em R\$ milhões) }\end{array}$ \\
\hline Cap & $\overline{\text { US\$ } 1.200 \text { milhões }}$ & Dezembro de 2004 & 6 \\
\hline Floor & US\$ 850 milhões & Dezembro de 2004 & (13) \\
\hline \multirow[t]{2}{*}{ Swap } & US\$ 125 milhões & Outubro de 2007 & (8) \\
\hline & & & (15) \\
\hline
\end{tabular}

A perda não realizada de $R \$ 15$ milhões representa o valor atual a pagar caso todas as operações fossem liquidadas em 31/12/00.

\section{CIA. 215}

19. Concentração de Risco de Crédito

A Companhia está potencialmente sujeita a risco de crédito com relação a suas aplicações financeiras, garantias dadas a bancos para financiar os clientes (operações de vendor) e contratos de swap. A Companhia limita seu risco efetuando aplicações financeiras ou firmando contratos de swap com o Banco " $X$ " ou outras instituições de primeira linha. Com relação às contas a receber e garantias fornecidas para os bancos que financiam os clientes (operações de vendor), a Companhia analisa e avalia o crédito dos clientes e, quando julga necessário, obtém cartas de crédito ou outras garantias não reais. Adicionalmente, a maioria das exportações é efetuada para os Estados Unidos e para a Europa e são seguradas por cartas de crédito. As despesas com créditos de liquidação duvidosa totalizam $R \$ 1.044$ mil em 2000 e $R \$ 4.713$ mil em 1999.

No tocante à divulgação quantitativa de risco, para surpresa, uma companhia ou $0,52 \%$ do total presta a referida informação. Uma instituição bancária (CIA. 46) divulga qual o montante do seu patrimônio líquido exigido - PLE para fazer face a fatores de risco de mercado de taxa de câmbio e de preço da commodity ouro (Resolução CMN no 2606), e de taxa de juros (Resolução CMN no 2692). Divulga também o VAR - Value at Risk para taxa de juros.

\section{CIA. 46}

Em relação à exigência de capital referente a riscos de mercado, a Resolução CMN no 2.606, de 27.05.1999, estabeleceu limites e exigência de capital relacionada à exposição em moedas estrangeiras e ouro. O Banco prosseguiu com a estratégia de redução dessa exposição, atingindo no corrente ano o objetivo de não alocar capital relacionado à exposição cambial. Por sua vez, a 
Resolução CMN no 2.692, de 24.02.2000, estabeleceu os critérios para exigência de capital para operações com taxas de juros prefixadas. O com- portamento ao longo do ano, referente à exigência de capital para riscos de mercado, pode ser observado na tabela abaixo:

Em R\$ milhões

\begin{tabular}{|l|r|r|r|r|}
\hline & \multicolumn{2}{|c|}{ Res. 2.606 } & \multicolumn{2}{c|}{ Res. 2.692 } \\
\hline & EXPOSIÇÃO & PLE $^{*}$ & V @ R & PLE \\
\hline Mínimo & 317 & & 52 & 122 \\
\hline Médio & 1.231 & 136 & 101 & 187 \\
\hline Máximo & 4.209 & 1.377 & 175 & 261 \\
\hline 31.12 .2000 & 708 & & 106 & 261 \\
\hline
\end{tabular}

Fonte: Cia. 46

* PLE: Patrimônio Líquido Exigido

No âmbito técnico, o Banco avançou no desenvolvimento e na integração do software de gestão de riscos de mercado, visando à sua utilização para a gestão de ativos e passivos. Nesse sentido, a ferramenta implantada no Banco permite não só a mensuração dos riscos de mercado pela técnica de V@R e os cálculos de sensibilidades e de "stress", já consagrados pela indústria financeira, mas também modernas técnicas de simulação dinâmica do balanço e de estruturação de hedge, que possibilitam melhor posicionamento da estrutura de ativos e passivos.

Com relação à estratégia operacional, 97 empresas ou $50,52 \%$ divulgam em nota explicativa que seu propósito em operações com esses instrumentos é hedge; o restante, 95 ou 49,48\%, não revela a estratégia adotada. Ao que parece, não haveria companhias envolvidas em operações de risco.

É um aspecto da pesquisa que chama a atenção. Uma primeira ilação que se faz é a de que possivelmente algumas companhias que, por vezes, ou corriqueiramente, enveredem em operações especulativas teriam resistência em revelar tal fato em nota explicativa.
Em se tratando do fair value dos instrumentos financeiros, somente 23 companhias ou $11,98 \%$ divulgam-no em nota explicativa, assim como as premissas utilizadas para seu cômputo. Muitas sentenças do tipo "o valor de mercado dos instrumentos financeiros não difere muito do seu valor contábil" ou "os cálculos realizados revelaram não haver diferenças significativas entre o valor de mercado e o valor contábil dos instrumentos financeiros" foram observadas sistematicamente.

Exercendo-se o senso crítico, não poderia uma debênture (um instrumento financeiro considerado por algumas companhias com valor de mercado igual ao valor contábil) que foi emitida há 3 anos, com seus termos refletindo as conjunturas econômicas da época, possuir o mesmo valor presente com os patamares de juros atuais. Ora, o modelo de avaliação de fluxo de caixa descontado é extremamente sensível a mudanças em suas variáveis, em especial às taxas de juros.

No que concerne à distribuição das companhias que transacionam instrumentos financeiros, pelos seus respectivos auditores independentes, o quadro é a seguir apresentado: 


\begin{tabular}{|c|c|c|c|c|c|}
\hline & Bancária & DR's & Ibovespa & Demais & \multirow[b]{6}{*}{$\Sigma$} \\
\hline A & 4 & 22 & 2 & 21 & \\
\hline B & 1 & 10 & 0 & 4 & \\
\hline C & 5 & 7 & 0 & 2 & \\
\hline D & 5 & 20 & 4 & 21 & \\
\hline \multirow{2}{*}{$\begin{array}{c}\text { E } \\
\text { "Big Five" }\end{array}$} & 1 & 5 & 3 & 8 & \\
\hline & 16 & 64 & 9 & 56 & \multirow[t]{4}{*}{145} \\
\hline $\mathrm{F}$ & 4 & 1 & 1 & 6 & \\
\hline G & 0 & 0 & 0 & 3 & \\
\hline $\mathrm{H}$ & 0 & 0 & 0 & 3 & \\
\hline \multirow{2}{*}{$\begin{array}{c}3 \text { maiores Brasil } \\
\text { demais }\end{array}$} & 4 & 1 & 1 & 12 & 18 \\
\hline & 11 & 0 & 0 & 18 & 29 \\
\hline Total das cias. & 31 & 65 & 10 & 86 & 192 \\
\hline
\end{tabular}

c/ intr. financ.

Fonte: Cia. 46

Para a pesquisa, o grupamento das companhias selecionadas por auditores independentes tem um certo grau de importância. A identificação de onde se concentra a maior parte dos resultados negativos da pesquisa permite chegar a algumas conclusões.
Computando-se os resultados obtidos com o cruzamento de dados de auditores independentes, com divulgação de risco (qualitativa e quantitativa), com divulgação de estratégia (arbitragem, hedging, e especulação) e com divulgação de fair value, tem-se que:

\begin{tabular}{|c|c|c|c|c|}
\hline & \multicolumn{2}{|c|}{ Divulga risco } & \multirow{2}{*}{$\begin{array}{l}\text { Divulga } \\
\text { Estratégia }\end{array}$} & \multirow{2}{*}{$\begin{array}{l}\text { Divulga } \\
\text { Fair Value }\end{array}$} \\
\hline & Qualitativo & Quantitativo & & \\
\hline A & 3 & 0 & 33 & 7 \\
\hline B & 1 & 0 & 7 & 0 \\
\hline C & 0 & 0 & 11 & 3 \\
\hline D & 1 & 0 & 22 & 5 \\
\hline$E$ & 0 & 0 & 11 & 3 \\
\hline "Big Five" & 5 & 0 & 84 & 18 \\
\hline $\mathrm{F}$ & 0 & 1 & 4 & 2 \\
\hline$G$ & 0 & 0 & 1 & 0 \\
\hline $\mathrm{H}$ & 0 & 0 & 0 & 0 \\
\hline 3 maiores Brasil & 0 & 1 & 5 & 2 \\
\hline demais & 0 & 0 & 8 & 3 \\
\hline Resultados positivos & 5 & 1 & 97 & 23 \\
\hline
\end{tabular}

Fonte: Cia. 46

A atuação das firmas de auditoria "big five" em mercados mundiais confere a elas tecnologia e expertise para avaliar a qualidade das informações prestadas ou não prestadas (quando seriam, por vezes, devidas) pelas companhias abertas brasileiras, com relação aos seus instrumentos financeiros. Essa consideração foi uma suposição inicial da pesquisa.

Contudo, tal aspecto não se refletiu nos resultados apurados. Do total de companhias auditadas pelas "big five", os resultados positivos foram 3,45\% para divulgação qualitativa de risco; 0,00\% para divulgação quantitativa de risco; $57,93 \%$ para divulgação de estratégia; $12,41 \%$ para divulgação de fair value.

Já para as três maiores firmas brasileiras de auditoria, os resultados positivos foram $0,00 \%$ para divulgação qualitativa de risco; $5,56 \%$ para divulgação quantitativa de risco; $27,78 \%$ para divulgação de estratégia; $11,11 \%$ para divulgação de fair value. 
Quanto às demais firmas de auditoria, os resultados positivos foram $0,00 \%$ para divulgação de risco qualitativa; $0,00 \%$ para divulgação de risco quantitativa; $27,59 \%$ para divulgação de estratégia; $10,34 \%$ para divulgação de fair value.

\section{CONCLUSÃO}

Restou evidenciado, de todo o exposto, que a resposta para a questão colocada para investigação acadêmica é não. A hipótese nula é extremamente válida. As companhias abertas brasileiras não prestam informações mínimas requeridas pela Instrução CVM no 235/95.

A responsabilidade por tal quadro deve ser imputada primariamente a administradores e contadores de companhias abertas brasileiras, pela atribuição de elaborarem as notas explicativas sobre instrumentos financeiros, anexas às demonstrações contábeis colocadas à disposição do público em geral.

Não há que se negar também uma parcela de responsabilidade do Órgão Regulador. Do ponto de vista da divulgação de risco (qualitativa ou quantitativa), a má qualidade observada no disclosure das companhias abertas brasileiras decorre substancialmente da ausência de regulamentação sobre a matéria.

Cumpre salientar nesse sentido que, no ano de 2002, foi expedido o Ofício Circular CVM/SEP/SNC/ no 01/2002, cujo item "3" serve ao propósito de apresentar um modelo não exaustivo de nota explicativa (anexo).
As causas para tal fato podem ser diversas. Embora não seja o propósito da pesquisa, visto que o problema delimitado inicialmente foi solucionado, supõe-se que as origens sejam:

(1) um possível despreparo dos profissionais brasileiros que lidam com a contabilidade das companhias abertas brasileiras, no tocante aos instrumentos financeiros;

(2) um possível despreparo dos profissionais brasileiros que lidam com a auditoria dessas companhias, no tocante aos instrumentos financeiros;

(3) uma possível resistência ao fair value accounting no Brasil, admitindo-se o preparo dos profissionais brasileiros (administradores e contadores de companhias abertas e auditores independentes);

(4) a pouca relevância dessas informações para os usuários das demonstrações contábeis no Brasil (sua passividade não permite outra conclusão); ou

(5) uma cultura incipiente no Brasil em matéria de instrumentos financeiros (pelo menos no tocante à sua importância em termos de informação para o mercado).

Espera-se que essas questões possam servir a estudos futuros, que encontrem as respostas para o quadro revelado. A expectativa que se tem é a de que este trabalho possa desencadear novas pesquisas, com foco na matéria, no âmbito acadêmico ou mesmo profissional, que desperte em estudiosos 0 interesse pelo seu aprofundamento. 


\section{REFERÊNCIAS BIBLIOGRÁFICAS}

CHANCE, Don M. Sobre a Divulgação do Risco de Derivativos: Injusta, Imprópria e Inconsistente. Tradução de: BM\&F. Resenha BM\&F, São Paulo, n. 116, [199-].

COMISSÃO DE VALORES MOBILIÁRIOS. Instrução CVM n. 235/95 de 23 de março de 1995. Dispõe sobre a divulgação, em nota explicativa, do valor de mercado dos instrumentos financeiros, reconhecidos ou não nas demonstrações financeiras de suas companhias. Diário Oficial da República Federativa do Brasil, Brasília, [1995].

COMISSÃO DE VALORES MOBILIÁRIOS. Nota Explicativa anexa à Instrução CVM n. 235/95 de 23 de março de 1995. Dispõe sobre a divulgação, em nota explicativa, do valor de mercado dos instrumentos financeiros, reconhecidos ou não nas demonstrações financeiras de suas companhias. Diário Oficial da República Federativa do Brasil, Brasília, [1995].

COMISSÃO DE VALORES MOBILIÁRIOS. Ofício Circular CVM/SNC/SEP/n. 04/95. Direcionado aos Auditores Independentes e Diretores de Relações com Investidores. Rio de Janeiro, 28 set. 1995.
COMISSÃO DE VALORES MOBILIÁRIOS. Ofício Circular CVM/SNC/SEP/n. 01/96. Direcionado aos Auditores Independentes e Diretores de Relações com Investidores. Rio de Janeiro, 03 jan. 1996.

COMISSÃO DE VALORES MOBILIÁRIOS. Ofício Circular CVM/SNC/SEP/n. 02/00. Direcionado aos Auditores Independentes e Diretores de Relações com Investidores. Rio de Janeiro, 29 dez. 2000.

COMISSÃO DE VALORES MOBILIÁRIOS. Ofício Circular CVM/SNC/SEP/n. 01/02. Direcionado aos Auditores Independentes e Diretores de Relações com Investidores. Rio de Janeiro, 14 jan. 2002.

DUARTE JR., Antônio Marcos et al. Gerenciamento de Riscos Corporativos: Classificação, Definições e Exemplos. Resenha $B M \& F$, São Paulo, n. 134, [199-].

STEVENSON, William J. Estatística Aplicada à Administração. São Paulo: Harbra, 1981. 


\section{APÊNDICE}

PLANILHA DE TRABALHO

\begin{tabular}{|c|c|c|c|c|c|c|c|}
\hline \multirow[b]{2}{*}{ Cia. } & \multirow{2}{*}{$\begin{array}{c}\text { Tem } \\
\text { Inst. Fin.? }\end{array}$} & \multicolumn{2}{|c|}{ Divulgação de Risco } & \multirow{2}{*}{$\frac{\text { Atuação no }}{\text { mercado }}$} & \multirow{2}{*}{$\begin{array}{c}\text { Divulga } \\
\text { fair value }\end{array}$} & \multirow{2}{*}{$\begin{array}{c}\text { Auditor } \\
\text { Indep. }\end{array}$} & \multirow{2}{*}{$\begin{array}{c}\text { Tipo de } \\
\text { instituição }\end{array}$} \\
\hline & & qualitativo & quantitativo & & & & \\
\hline 1 & SIM & NÃO & $N \tilde{O} O$ & HEDGING & NÃO & $D$ & IBOVESPA \\
\hline 2 & SIM & $N \tilde{O} O$ & $N \tilde{A O}$ & $N \tilde{O} O$ & $N \tilde{A O}$ & $H$ & DEMAIS \\
\hline 3 & SIM & $N \tilde{O} O$ & $N \tilde{A O}$ & $N \tilde{A O}$ & $N \tilde{A O}$ & $H$ & DEMAIS \\
\hline 4 & SIM & $N \tilde{A O}$ & $N \tilde{A O}$ & $N \tilde{A} O$ & $N \tilde{A O}$ & Outras & DEMAIS \\
\hline 5 & SIM & $N \tilde{O} O$ & $N \tilde{O} O$ & $N \tilde{O} O$ & $N \tilde{A O}$ & $D$ & DEMAIS \\
\hline 6 & SIM & $N \tilde{O} O$ & $N \tilde{A} O$ & HEDGING & $N \tilde{O} O$ & $C$ & DEMAIS \\
\hline 7 & SIM & $N \tilde{A} O$ & $N \tilde{A} O$ & $N \tilde{A O}$ & SIM & $D$ & IBOVESPA \\
\hline 8 & $S I M$ & $N \tilde{A} O$ & $N \tilde{A} O$ & $N \tilde{A} O$ & $N \tilde{A} O$ & $D$ & DEMAIS \\
\hline 9 & SIM & $N \tilde{A} O$ & $N \tilde{A O}$ & $N \tilde{O} O$ & $N \tilde{A O}$ & $E$ & DEMAIS \\
\hline 10 & SIM & $N \tilde{A} O$ & $N \tilde{A} O$ & $N \tilde{A O}$ & $N \tilde{A} O$ & $E$ & DEMAIS \\
\hline 11 & $N \tilde{A} O$ & $N \tilde{A} O$ & $N \tilde{A} O$ & $N \tilde{A O}$ & $N \tilde{A} O$ & $A$ & DEMAIS \\
\hline 12 & SIM & $N \tilde{A} O$ & $N \tilde{A} O$ & HEDGING & $N \tilde{A} O$ & $C$ & DEMAIS \\
\hline 13 & $N \tilde{A} O$ & $N \tilde{A O}$ & $N \tilde{A} O$ & $N \tilde{O} O$ & $N \tilde{O} O$ & $A$ & DEMAIS \\
\hline 14 & SIM & $N \tilde{A} O$ & $N \tilde{A} O$ & HEDGING & $N \tilde{A} O$ & $F$ & DEMAIS \\
\hline 15 & $N \tilde{A} O$ & $N \tilde{A O}$ & $N \tilde{O} O$ & $N \tilde{O} O$ & $N \tilde{O} O$ & $D$ & DEMAIS \\
\hline 16 & SIM & $N \tilde{A} O$ & $N \tilde{O} O$ & $N \tilde{O} O$ & $N \tilde{A O}$ & $F$ & DEMAIS \\
\hline 17 & $S I M$ & $N \tilde{A} O$ & $N \tilde{A} O$ & $N \tilde{A O}$ & $N \tilde{O} O$ & $A$ & DEMAIS \\
\hline 18 & SIM & $N \tilde{A} O$ & $N \tilde{O} O$ & HEDGING & SIM & $A$ & DEMAIS \\
\hline 19 & $N \tilde{A O}$ & $N \tilde{A} O$ & $N \tilde{A} O$ & $N \tilde{O} O$ & $N \tilde{A} O$ & $H$ & DEMAIS \\
\hline 20 & $N \tilde{A} O$ & $N \tilde{A} O$ & $N \tilde{O} O$ & $N \tilde{O} O$ & $N \tilde{A} O$ & $A$ & DEMAIS \\
\hline 21 & SIM & $N \tilde{A} O$ & $N \tilde{A} O$ & $N \tilde{A} O$ & $N \tilde{A} O$ & Outras & DEMAIS \\
\hline 22 & $N \tilde{A} O$ & $N \tilde{O} O$ & $N \tilde{A O}$ & $N \tilde{O} O$ & $N \tilde{A O}$ & Outras & DEMAIS \\
\hline 23 & SIM & $N \tilde{A} O$ & $N \tilde{A} O$ & HEDGING & $N \tilde{A} O$ & $E$ & DEMAIS \\
\hline 24 & SIM & $N \tilde{O} O$ & $N \tilde{O} O$ & $N \tilde{O} O$ & $N \tilde{O} O$ & $D$ & DEMAIS \\
\hline 25 & $N \tilde{A} O$ & $N \tilde{A} O$ & $N \tilde{A} O$ & $N \tilde{A O}$ & $N \tilde{A} O$ & Outras & DEMAIS \\
\hline 26 & $S I M$ & $N \tilde{A} O$ & $N \tilde{A} O$ & HEDGING & $S I M$ & $A$ & $D R ' S$ \\
\hline 27 & SIM & $N \tilde{A} O$ & $N \tilde{A} O$ & HEDGING & $N \tilde{A} O$ & $D$ & DEMAIS \\
\hline 28 & SIM & $N \tilde{A} O$ & $N \tilde{A} O$ & $N A \tilde{O}$ & $N \tilde{A} O$ & Outras & DEMAIS \\
\hline 29 & SIM & $N \tilde{A} O$ & $N \tilde{A} O$ & $N \tilde{A O}$ & $N \tilde{A} O$ & $D$ & DEMAIS \\
\hline 30 & SIM & $N \tilde{A} O$ & $N \tilde{A} O$ & HEDGING & $N \tilde{A} O$ & $D$ & DEMAIS \\
\hline 31 & $N \tilde{A} O$ & $N \tilde{A} O$ & $N \hat{A} O$ & $N \tilde{A} O$ & $N \tilde{A} O$ & $A$ & DEMAIS \\
\hline 32 & $S I M$ & $N \tilde{A} O$ & $N A \tilde{O}$ & $N \tilde{A O}$ & $N \tilde{A} O$ & Outras & DEMAIS \\
\hline 33 & $S I M$ & $N \tilde{A} O$ & $N \tilde{A} O$ & $N \tilde{A} O$ & $N \tilde{A} O$ & $F$ & DEMAIS \\
\hline 34 & SIM & $N \tilde{A} O$ & $N \tilde{A} O$ & HEDGING & $N \tilde{A O}$ & $A$ & DEMAIS \\
\hline 35 & SIM & $N \tilde{A} O$ & $N A \tilde{O}$ & $N \tilde{A} O$ & $N \tilde{A} O$ & $A$ & DEMAIS \\
\hline 36 & $S I M$ & $N \tilde{A} O$ & $N \hat{A} O$ & HEDGING & $N \tilde{A} O$ & Outras & DEMAIS \\
\hline 37 & SIM & $N \tilde{A} O$ & $N A \tilde{O}$ & HEDGING & $N \tilde{A} O$ & $C$ & $D R ' S$ \\
\hline 38 & $S I M$ & $N \tilde{A} O$ & $N \hat{A} O$ & HEDGING & $N \tilde{A} O$ & $C$ & BANCÁRIA \\
\hline 39 & SIM & $N \tilde{A} O$ & $N \tilde{A} O$ & $N \tilde{A O}$ & $N \tilde{A} O$ & Outras & BANCÁRIA \\
\hline 40 & SIM & $N \tilde{A} O$ & $N \hat{A} O$ & HEDGING & $N \tilde{A} O$ & $F$ & BANCÁRIA \\
\hline 41 & SIM & $N \tilde{A} O$ & $N A \tilde{O}$ & $N \tilde{A O}$ & $N \tilde{A} O$ & $D$ & BANCÁRIA \\
\hline
\end{tabular}




\section{APÊNDICE}

PLANILHA DE TRABALHO

\begin{tabular}{|c|c|c|c|c|c|c|c|}
\hline \multirow[b]{2}{*}{ Cia. } & \multirow{2}{*}{$\frac{\text { Tem }}{\text { Inst. Fin.? }}$} & \multicolumn{2}{|c|}{ Divulgação de Risco } & \multirow{2}{*}{$\frac{\text { Atuação no }}{\text { mercado }}$} & \multirow{2}{*}{$\begin{array}{c}\text { Divulga } \\
\text { fair value }\end{array}$} & \multirow{2}{*}{$\begin{array}{c}\text { Auditor } \\
\text { Indep. }\end{array}$} & \multirow{2}{*}{$\begin{array}{c}\text { Tipo de } \\
\text { instituição }\end{array}$} \\
\hline & & qualitativo & quantitativo & & & & \\
\hline 42 & SIM & NÃO & $N \tilde{A} O$ & HEDGING & SIM & $D$ & BANCÁRIA \\
\hline 43 & SIM & $N \tilde{A O}$ & NÃO & HEDGING & $N \tilde{A O}$ & $C$ & BANCÁRIA \\
\hline 44 & SIM & $N \tilde{A} O$ & NÃO & HEDGING & $N \tilde{A O}$ & $D$ & BANCÁRIA \\
\hline 45 & SIM & NÃO & NÃO & HEDGING & SIM & $A$ & BANCÁRIA \\
\hline 46 & SIM & NÃO & SIM & HEDGING & SIM & $F$ & BANCÁRIA \\
\hline 47 & SIM & NÃO & $N \tilde{A} O$ & $N \tilde{A} O$ & $N \tilde{A O}$ & Outras & BANCÁRIA \\
\hline 48 & SIM & NÃO & NÃO & HEDGING & NÃO & $D$ & BANCÁRIA \\
\hline 49 & SIM & $N \tilde{A O}$ & $N \tilde{A} O$ & HEDGING & $N \tilde{A O}$ & Outras & BANCÁRIA \\
\hline 50 & SIM & $N \tilde{A O}$ & $N \tilde{O} O$ & HEDGING & SIM & Outras & BANCÁRIA \\
\hline 51 & SIM & $N \tilde{A} O$ & $N \tilde{A} O$ & $N \tilde{A} O$ & $N \tilde{A O}$ & $E$ & BANCÁRIA \\
\hline 52 & SIM & NÃO & NÂO & $N \tilde{O} O$ & NÂO & Outras & BANCÁRIA \\
\hline 53 & SIM & $N \tilde{A} O$ & $N \tilde{A} O$ & HEDGING & $N \tilde{A O}$ & Outras & BANCÁRIA \\
\hline 54 & SIM & NÃO & $N \tilde{O} O$ & HEDGING & NÃO & $C$ & BANCÁRIA \\
\hline 55 & SIM & $N \tilde{A} O$ & $N \tilde{A} O$ & $N \tilde{A} O$ & $S I M$ & Outras & BANCÁRIA \\
\hline 56 & SIM & NÃO & $N \tilde{O} O$ & HEDGING & NÃO & $B$ & BANCÁRIA \\
\hline 57 & SIM & $N \tilde{A O}$ & $N \tilde{O} O$ & $N \tilde{A O}$ & $N \tilde{A} O$ & Outras & BANCÁRIA \\
\hline 58 & SIM & NÃO & $N \tilde{O} O$ & $N \tilde{O} O$ & $N \tilde{A} O$ & Outras & BANCÁRIA \\
\hline 59 & SIM & $N \tilde{A O}$ & $N \tilde{O} O$ & $N \tilde{A O}$ & $N \tilde{A} O$ & Outras & DEMAIS \\
\hline 60 & SIM & $N A \tilde{O}$ & $N \tilde{A} O$ & HEDGING & SIM & $C$ & BANCÁRIA \\
\hline 61 & SIM & $N \tilde{A} O$ & $N \tilde{A} O$ & HEDGING & SIM & Outras & BANCÁRIA \\
\hline 62 & SIM & $N \tilde{A} O$ & $N \tilde{O} O$ & HEDGING & $N \tilde{A} O$ & Outras & BANCÁRIA \\
\hline 63 & SIM & $N \tilde{A} O$ & $N A \tilde{O}$ & $N A \tilde{O}$ & NÃO & $F$ & BANCÁRIA \\
\hline 64 & SIM & $N \tilde{A O}$ & $N \tilde{O} O$ & HEDGING & SIM & $D$ & BANCÁRIA \\
\hline 65 & SIM & $N \tilde{A O}$ & $N A \tilde{O}$ & HEDGING & NÃO & $E$ & DEMAIS \\
\hline 66 & SIM & $N \tilde{A O}$ & $N \tilde{A} O$ & $N \tilde{A} O$ & NÃO & $D$ & DEMAIS \\
\hline 67 & SIM & $N \tilde{A} O$ & $N \tilde{A} O$ & HEDGING & $N \tilde{A} O$ & $E$ & DEMAIS \\
\hline 68 & SIM & $N \tilde{A} O$ & NÃO & HEDGING & $N \tilde{A O}$ & $A$ & DEMAIS \\
\hline 69 & SIM & $N \tilde{A} O$ & $N \tilde{A O}$ & $N \tilde{A} O$ & $N \tilde{A} O$ & Outras & DEMAIS \\
\hline 70 & SIM & $N \tilde{A O}$ & $N \tilde{A O}$ & $N \tilde{A O}$ & $N \tilde{A O}$ & Outras & DEMAIS \\
\hline 71 & SIM & $N \tilde{A} O$ & $N \tilde{A} O$ & HEDGING & $N \tilde{A} O$ & Outras & DEMAIS \\
\hline 72 & SIM & $N \tilde{A} O$ & $N \tilde{A} O$ & $N A \tilde{O}$ & $N \tilde{A} O$ & $B$ & DEMAIS \\
\hline 73 & $N \tilde{A} O$ & $N \tilde{A} O$ & $N A \tilde{O}$ & $N \tilde{A} O$ & $N \tilde{A} O$ & Outras & DEMAIS \\
\hline 74 & SIM & $N \tilde{A} O$ & $N \tilde{A} O$ & $N A \tilde{O}$ & $N \tilde{A} O$ & $F$ & DEMAIS \\
\hline 75 & SIM & $N \tilde{A} O$ & $N \tilde{A} O$ & $N A \tilde{O}$ & $N \tilde{A} O$ & $A$ & DEMAIS \\
\hline 76 & SIM & $N \tilde{A} O$ & $N \tilde{A} O$ & HEDGING & $N \tilde{A} O$ & $A$ & BANCÁRIA \\
\hline 77 & SIM & $N \tilde{A} O$ & $N \tilde{A} O$ & HEDGING & $N \tilde{A} O$ & $D$ & DEMAIS \\
\hline 78 & $N \tilde{A} O$ & $N \tilde{A} O$ & $N \tilde{A} O$ & $N \tilde{A} O$ & $N \tilde{A} O$ & $H$ & DEMAIS \\
\hline 79 & $N \tilde{A} O$ & $N \tilde{A} O$ & $N \tilde{A} O$ & $N A \tilde{O}$ & $N \tilde{A} O$ & Outras & DEMAIS \\
\hline 80 & SIM & $N \tilde{A} O$ & $N \tilde{A} O$ & $N \hat{A} O$ & $N \tilde{A} O$ & Outras & DEMAIS \\
\hline 81 & SIM & $N \tilde{A} O$ & $N \tilde{A} O$ & HEDGING & $N \tilde{A} O$ & $G$ & DEMAIS \\
\hline 82 & SIM & $N \tilde{A O}$ & $N A \tilde{O}$ & HEDGING & $N A \tilde{O}$ & $A$ & DEMAIS \\
\hline
\end{tabular}




\section{APÊNDICE}

PLANILHA DE TRABALHO

\begin{tabular}{|c|c|c|c|c|c|c|c|}
\hline & Tem & Divulgaç & de Risco & Atuação no & Divulga & Auditor & Tipo de \\
\hline Cia. & Inst. Fin.? & qualitativo & quantitativo & mercado & fair value & Indep. & instituição \\
\hline 83 & $S I M$ & $N \tilde{O} O$ & $N \tilde{O} O$ & HEDGING & $N \tilde{O} O$ & $A$ & DEMAIS \\
\hline 84 & SIM & $N \tilde{A O}$ & $N \tilde{A O}$ & HEDGING & $N \tilde{A O}$ & Outras & DEMAIS \\
\hline 85 & $N \tilde{A} O$ & $N \tilde{A} O$ & $N \tilde{A} O$ & $N \tilde{A} O$ & $N \tilde{O} O$ & $C$ & DEMAIS \\
\hline 86 & SIM & $N \tilde{A O}$ & $N \tilde{A} O$ & HEDGING & $N \tilde{O} O$ & $D$ & DEMAIS \\
\hline 87 & SIM & $N \tilde{O} O$ & $N \tilde{O} O$ & $N \tilde{A} O$ & $N \tilde{O} O$ & $G$ & DEMAIS \\
\hline 88 & SIM & $N \tilde{O} O$ & $N \tilde{A O}$ & $N \tilde{O} O$ & $N \tilde{O} O$ & $D$ & DEMAIS \\
\hline 89 & $S I M$ & $N \tilde{A} O$ & $N \tilde{A O}$ & HEDGING & SIM & $E$ & $D R ' S$ \\
\hline 90 & $S I M$ & $N \tilde{A} O$ & $N \tilde{A} O$ & HEDGING & $N \tilde{A} O$ & $B$ & $D R ' S$ \\
\hline 91 & SIM & $N \tilde{O} O$ & $N \tilde{A} O$ & $N \tilde{O} O$ & $N \tilde{O} O$ & $D$ & DEMAIS \\
\hline 92 & $S I M$ & $N \tilde{A} O$ & $N \tilde{A} O$ & $N \tilde{A} O$ & $N \tilde{A} O$ & $H$ & DEMAIS \\
\hline 93 & SIM & $N \tilde{A} O$ & $N \tilde{A O}$ & $N \tilde{O} O$ & $N \tilde{O} O$ & $D$ & DEMAIS \\
\hline 94 & $S I M$ & $N \tilde{A} O$ & $N \tilde{A} O$ & HEDGING & $N \tilde{A} O$ & $A$ & DEMAIS \\
\hline 95 & SIM & SIM & $N \tilde{O} O$ & HEDGING & $N \tilde{O} O$ & $A$ & $D R ' S$ \\
\hline 96 & SIM & SIM & $N \tilde{A} O$ & HEDGING & $N \tilde{O} O$ & $A$ & DEMAIS \\
\hline 97 & SIM & $N \tilde{O} O$ & $N \tilde{A} O$ & HEDGING & $N \tilde{O} O$ & $E$ & DEMAIS \\
\hline 98 & $S I M$ & $N \tilde{A} O$ & $N \tilde{A} O$ & HEDGING & $N \tilde{A} O$ & $A$ & DEMAIS \\
\hline 99 & $S I M$ & $N \tilde{A} O$ & $N \tilde{A} O$ & HEDGING & $N \tilde{O} O$ & $B$ & DEMAIS \\
\hline 100 & $S I M$ & $N \tilde{A} O$ & $N \tilde{A} O$ & $N \tilde{A} O$ & $N \tilde{A} O$ & $D$ & DEMAIS \\
\hline 101 & SIM & $N \tilde{A} O$ & $N \tilde{A} O$ & $N \tilde{O} O$ & $N \tilde{O} O$ & $D$ & DEMAIS \\
\hline 102 & $N \tilde{A} O$ & $N \tilde{A} O$ & $N \tilde{A} O$ & $N \tilde{A} O$ & $N \tilde{A} O$ & $D$ & $D R^{\prime} S$ \\
\hline 103 & SIM & $N \tilde{A O}$ & $N \tilde{A} O$ & $N \tilde{O} O$ & SIM & $F$ & BANCÁRIA \\
\hline 104 & $S I M$ & $N \tilde{A} O$ & $N \tilde{A} O$ & $N \tilde{A} O$ & $N \tilde{A} O$ & Outras & DEMAIS \\
\hline 105 & SIM & $N \tilde{A O}$ & $N \tilde{A} O$ & HEDGING & SIM & $E$ & DEMAIS \\
\hline 106 & SIM & $N \tilde{A} O$ & $N \tilde{A} O$ & HEDGING & SIM & $A$ & DEMAIS \\
\hline 107 & SIM & $N \tilde{A} O$ & $N \tilde{A} O$ & HEDGING & $N \tilde{O} O$ & $A$ & DEMAIS \\
\hline 108 & $N \tilde{A} O$ & $N \tilde{A} O$ & $N \tilde{A} O$ & $N \tilde{A O}$ & $N \tilde{A O}$ & Outras & DEMAIS \\
\hline 109 & $N \tilde{O} O$ & $N \tilde{O} O$ & $N \tilde{O} O$ & $N \tilde{O} O$ & $N \tilde{O} O$ & $E$ & DEMAIS \\
\hline 110 & $N \tilde{A} O$ & $N \tilde{A O}$ & $N \tilde{A} O$ & $N \tilde{O} O$ & $N \tilde{A O}$ & Outras & DEMAIS \\
\hline 111 & $N \tilde{A} O$ & $N \tilde{A O}$ & $N \tilde{A} O$ & $N \tilde{O} O$ & $N \tilde{O} O$ & $H$ & DEMAIS \\
\hline 112 & SIM & $N \tilde{A O}$ & $N \tilde{A} O$ & $N \tilde{O} O$ & $N \tilde{A O}$ & $A$ & DEMAIS \\
\hline 113 & $N \tilde{A} O$ & $N \tilde{A} O$ & $N \tilde{A} O$ & $N \tilde{A O}$ & $N \tilde{O} O$ & $H$ & DEMAIS \\
\hline 114 & SIM & $N \tilde{A} O$ & $N \tilde{A} O$ & HEDGING & $N \tilde{O} O$ & $A$ & DEMAIS \\
\hline 115 & SIM & $N \tilde{O} O$ & $N \tilde{A O}$ & $N \tilde{O} O$ & $N \tilde{A O}$ & Outras & DEMAIS \\
\hline 116 & SIM & $N \tilde{O} O$ & $N \tilde{A} O$ & $N \tilde{O} O$ & $N \tilde{O} O$ & $D$ & DEMAIS \\
\hline 117 & SIM & $N \tilde{O} O$ & $N \tilde{A} O$ & $N \tilde{A O}$ & $N \tilde{A O}$ & Outras & DEMAIS \\
\hline 118 & SIM & $N \tilde{O} O$ & $N \tilde{A} O$ & $N \tilde{O} O$ & $N \tilde{A} O$ & $A$ & $D R^{\prime} S$ \\
\hline 119 & SIM & $N \tilde{O} O$ & $N \tilde{A} O$ & HEDGING & $N \tilde{O} O$ & $E$ & DEMAIS \\
\hline 120 & SIM & $N \tilde{O} O$ & $N \tilde{A} O$ & $N \tilde{O} O$ & $N \tilde{A} O$ & $B$ & $D R^{\prime} S$ \\
\hline 121 & SIM & $N \tilde{O} O$ & $N \tilde{A} O$ & $N \tilde{O} O$ & $N \tilde{O} O$ & $D$ & DEMAIS \\
\hline 122 & SIM & $N \tilde{A} O$ & $N \tilde{A O}$ & $N \tilde{O} O$ & $N \tilde{A O}$ & $D$ & $D R ' S$ \\
\hline 123 & $S I M$ & $N \tilde{A} O$ & $N \tilde{A} O$ & $N \tilde{A} O$ & $N \tilde{A O}$ & $B$ & DEMAIS \\
\hline
\end{tabular}




\section{APÊNDICE}

PLANILHA DE TRABALHO

\begin{tabular}{|c|c|c|c|c|c|c|c|}
\hline \multirow[b]{2}{*}{ Cia. } & \multirow{2}{*}{$\frac{\text { Tem }}{\text { Inst. Fin.? }}$} & \multicolumn{2}{|c|}{ Divulgação de Risco } & \multirow{2}{*}{$\begin{array}{c}\text { Atuação no } \\
\text { mercado }\end{array}$} & \multirow{2}{*}{$\begin{array}{c}\text { Divulga } \\
\text { fair value }\end{array}$} & \multirow{2}{*}{$\begin{array}{c}\text { Auditor } \\
\text { Indep. }\end{array}$} & \multirow{2}{*}{$\frac{\text { Tipo de }}{\text { instituição }}$} \\
\hline & & qualitativo & quantitativo & & & & \\
\hline 124 & $S I M$ & $N \tilde{A} O$ & $N \tilde{A} O$ & HEDGING & $N \tilde{O} O$ & $A$ & DEMAIS \\
\hline 125 & SIM & $N \tilde{O} O$ & $N \tilde{A O}$ & $N \tilde{O} O$ & $N \tilde{A O}$ & Outras & DEMAIS \\
\hline 126 & $N \tilde{O} O$ & $N \tilde{O} O$ & $N \tilde{O} O$ & $N \tilde{O} O$ & $N \tilde{A O}$ & Outras & DEMAIS \\
\hline 127 & SIM & $N \tilde{A O}$ & $N \tilde{O} O$ & HEDGING & $N \tilde{A O}$ & $A$ & $D R ' S$ \\
\hline 128 & SIM & $N \tilde{A O}$ & $N \tilde{A O}$ & HEDGING & $N \tilde{A O}$ & $A$ & DEMAIS \\
\hline 129 & SIM & $N \tilde{A O}$ & $N \tilde{A O}$ & HEDGING & $N \tilde{O} O$ & $A$ & $D R^{\prime} S$ \\
\hline 130 & SIM & $N \tilde{A O}$ & $N \tilde{O} O$ & HEDGING & $N \tilde{O} O$ & $E$ & $D R^{\prime} S$ \\
\hline 131 & SIM & $N \tilde{O} O$ & $N \tilde{O} O$ & $N \tilde{O} O$ & $N \tilde{O} O$ & Outras & DEMAIS \\
\hline 132 & SIM & $N \tilde{O} O$ & $N \tilde{O} O$ & HEDGING & $N \tilde{A O}$ & $D$ & DEMAIS \\
\hline 133 & SIM & $N \tilde{O} O$ & $N \tilde{O} O$ & $N \tilde{O} O$ & $N \tilde{O} O$ & $A$ & DEMAIS \\
\hline 134 & $N \tilde{A} O$ & $N \tilde{A} O$ & $N \tilde{A} O$ & $N \tilde{O} O$ & $N \tilde{A O}$ & $F$ & DEMAIS \\
\hline 135 & SIM & $N \tilde{O} O$ & $N \tilde{O} O$ & $N \tilde{O} O$ & $N \tilde{O} O$ & $D$ & $D R^{\prime} S$ \\
\hline 136 & SIM & $N \tilde{A} O$ & $N \tilde{A} O$ & $N \tilde{O} O$ & $N \tilde{A} O$ & $G$ & DEMAIS \\
\hline 137 & SIM & $N \tilde{O} O$ & $N \tilde{O} O$ & $N \tilde{O} O$ & $N \tilde{O} O$ & $F$ & DEMAIS \\
\hline 138 & SIM & $N \tilde{A} O$ & $N \tilde{O} O$ & $N \tilde{O} O$ & $N \tilde{A} O$ & $D$ & DEMAIS \\
\hline 139 & SIM & $N \tilde{A} O$ & $N \tilde{O} O$ & $N \tilde{O} O$ & $N \tilde{O} O$ & $D$ & DEMAIS \\
\hline 140 & SIM & $N \tilde{O} O$ & $N \tilde{O} O$ & $N \tilde{O} O$ & $N \tilde{O} O$ & $E$ & $D R^{\prime} S$ \\
\hline 141 & $N \tilde{A} O$ & $N \tilde{A O}$ & $N \tilde{A O}$ & $N \tilde{O} O$ & $N \tilde{A} O$ & Outras & DEMAIS \\
\hline 142 & SIM & $N \tilde{O} O$ & $N \tilde{O} O$ & $N \tilde{O} O$ & $N \tilde{O} O$ & $D$ & $D R^{\prime} S$ \\
\hline 143 & SIM & $N \tilde{A} O$ & $N \tilde{O} O$ & $N \tilde{O} O$ & $N \tilde{A} O$ & $D$ & DEMAIS \\
\hline 144 & SIM & $N \tilde{A} O$ & $N \tilde{A} O$ & $N \tilde{A} O$ & $N \tilde{A} O$ & $A$ & IBOVESPA \\
\hline 145 & SIM & $N \tilde{A O}$ & $N \tilde{O} O$ & $N \tilde{O} O$ & $N \tilde{O} O$ & $A$ & $D R ' S$ \\
\hline 146 & SIM & $N \tilde{A} O$ & $N \tilde{A} O$ & HEDGING & SIM & $D$ & $D R^{\prime} S$ \\
\hline 147 & SIM & $N \tilde{O} O$ & $N \tilde{O} O$ & HEDGING & SIM & $A$ & IBOVESPA \\
\hline 148 & SIM & $N \tilde{A} O$ & $N \tilde{A} O$ & $N \tilde{A} O$ & SIM & $C$ & $D R^{\prime} S$ \\
\hline 149 & SIM & $N \tilde{O} O$ & $N \tilde{O} O$ & HEDGING & $N \tilde{A} O$ & $D$ & $D R ' S$ \\
\hline 150 & SIM & $N \tilde{A} O$ & $N \tilde{A} O$ & $N \tilde{A O}$ & $N \tilde{A O}$ & $E$ & $D R^{\prime} S$ \\
\hline 151 & SIM & SIM & $N \tilde{O} O$ & HEDGING & $N \tilde{O} O$ & $A$ & $D R ' S$ \\
\hline 152 & SIM & $N \tilde{A O}$ & $N \tilde{A} O$ & $N \tilde{A} O$ & $N \tilde{A O}$ & $B$ & $D R^{\prime} S$ \\
\hline 153 & SIM & $N \tilde{O} O$ & $N \tilde{O} O$ & $N \tilde{O} O$ & $N \tilde{O} O$ & Outras & DEMAIS \\
\hline 154 & SIM & $N \tilde{O} O$ & $N \tilde{O} O$ & $N \tilde{O} O$ & $N \tilde{O} O$ & Outras & DEMAIS \\
\hline 155 & SIM & NÃO & $N \tilde{O} O$ & HEDGING & $N \tilde{O} O$ & $D$ & $D R ' S$ \\
\hline 156 & SIM & $N \tilde{A} O$ & NÃO & $N \tilde{O} O$ & $N \tilde{O} O$ & $C$ & $D R^{\prime} S$ \\
\hline 157 & SIM & $N \tilde{A O}$ & $N \tilde{A O}$ & $N \tilde{O} O$ & $N \tilde{A O}$ & $A$ & DEMAIS \\
\hline 158 & SIM & $N \tilde{A} O$ & $N \tilde{A O}$ & $N \tilde{A O}$ & $N \tilde{A} O$ & $D$ & DEMAIS \\
\hline 159 & SIM & $N \tilde{O} O$ & $N \tilde{A O}$ & HEDGING & $N \tilde{A O}$ & $E$ & IBOVESPA \\
\hline 160 & SIM & $N \tilde{O} O$ & $N \tilde{O} O$ & $N \tilde{O} O$ & $N \tilde{O} O$ & $B$ & DEMAIS \\
\hline 161 & SIM & $N \tilde{A O}$ & $N \tilde{A O}$ & HEDGING & $N \tilde{A O}$ & $D$ & $D R^{\prime} S$ \\
\hline 162 & SIM & $N \tilde{A} O$ & $N \tilde{A O}$ & $N \tilde{O} O$ & $N \tilde{A O}$ & $D$ & $D R^{\prime} S$ \\
\hline 163 & SIM & $N \tilde{A O}$ & $N \tilde{A} O$ & $N \tilde{A O}$ & $N \tilde{A O}$ & $D$ & $D R^{\prime} S$ \\
\hline 164 & SIM & $N \tilde{A O}$ & $N \tilde{O} O$ & $N \tilde{O} O$ & $N \tilde{A O}$ & $B$ & $D R ' S$ \\
\hline
\end{tabular}




\section{APÊNDICE}

PLANILHA DE TRABALHO

\begin{tabular}{|c|c|c|c|c|c|c|c|}
\hline & Tem & Divulgaç & de Risco & Atuação no & Divulga & Auditor & Tipo de \\
\hline Cia. & Inst. Fin.? & qualitativo & quantitativo & mercado & fair value & Indep. & instituição \\
\hline 165 & $S I M$ & $N \tilde{O} O$ & $N \tilde{O} O$ & $N \tilde{O} O$ & $N \tilde{O} O$ & $D$ & $D R ' S$ \\
\hline 166 & SIM & $N \tilde{A O}$ & $N \tilde{A O}$ & $N \tilde{O} O$ & $N \tilde{A O}$ & $B$ & $D R^{\prime} S$ \\
\hline 167 & SIM & $N \tilde{A O}$ & $N \tilde{A O}$ & HEDGING & $N \tilde{O} O$ & $B$ & $D R^{\prime} S$ \\
\hline 168 & SIM & $N \tilde{A O}$ & $N \tilde{A O}$ & HEDGING & $N \tilde{A O}$ & $A$ & $D R^{\prime} S$ \\
\hline 169 & SIM & $N \tilde{O} O$ & $N \tilde{O} O$ & $N \tilde{A} O$ & $N \tilde{O} O$ & $A$ & DEMAIS \\
\hline 170 & SIM & $N \tilde{O} O$ & $N \tilde{A} O$ & $N \tilde{A O}$ & $N \tilde{A O}$ & $A$ & DEMAIS \\
\hline 171 & $N \tilde{A} O$ & $N \tilde{A} O$ & $N \tilde{A} O$ & $N \tilde{A O}$ & $N \tilde{A} O$ & Outras & IBOVESPA \\
\hline 172 & SIM & $N \tilde{A} O$ & $N \tilde{A} O$ & $N \tilde{A} O$ & $N \tilde{A} O$ & $A$ & $D R^{\prime} S$ \\
\hline 173 & SIM & $N \tilde{A} O$ & $N \tilde{A O}$ & $N \tilde{O} O$ & $N \tilde{O} O$ & $D$ & IBOVESPA \\
\hline 174 & $S I M$ & $N \tilde{A} O$ & $N \tilde{A} O$ & HEDGING & $S I M$ & $C$ & BANCÁRIA \\
\hline 175 & SIM & $N \tilde{A} O$ & $N \tilde{A} O$ & $N \tilde{A O}$ & $N \tilde{O} O$ & $D$ & $D R ' S$ \\
\hline 176 & $S I M$ & $N \tilde{A} O$ & $N \tilde{A} O$ & $N \tilde{A} O$ & $N \tilde{A} O$ & $A$ & $D R^{\prime} S$ \\
\hline 177 & SIM & $N \tilde{A O}$ & $N \tilde{O} O$ & NÃO & $N \tilde{O} O$ & $F$ & DEMAIS \\
\hline 178 & $S I M$ & $N \tilde{A} O$ & $N \tilde{A} O$ & HEDGING & SIM & $E$ & IBOVESPA \\
\hline 179 & SIM & $N \tilde{A O}$ & $N \tilde{A} O$ & HEDGING & $N \tilde{O} O$ & $A$ & $D R ' S$ \\
\hline 180 & $S I M$ & $N \tilde{A} O$ & $N \tilde{A} O$ & $N \tilde{A} O$ & $N \tilde{A} O$ & $F$ & $D R^{\prime} S$ \\
\hline 181 & $S I M$ & $N \tilde{A} O$ & $N \tilde{A} O$ & $N \tilde{O} O$ & $N \tilde{O} O$ & $A$ & $D R ' S$ \\
\hline 182 & $S I M$ & $N \tilde{A} O$ & $N \tilde{A} O$ & HEDGING & $N \tilde{A} O$ & $C$ & $D R ' S$ \\
\hline 183 & SIM & $N \tilde{A} O$ & $N \tilde{A} O$ & HEDGING & $N \tilde{O} O$ & $D$ & $D R ' S$ \\
\hline 184 & $S I M$ & $N \tilde{A} O$ & $N \tilde{A} O$ & HEDGING & $N \tilde{A} O$ & $A$ & $D R^{\prime} S$ \\
\hline 185 & SIM & $N \tilde{A} O$ & $N \tilde{O} O$ & HEDGING & $N \tilde{O} O$ & $D$ & $D R ' S$ \\
\hline 186 & $S I M$ & $N \tilde{A} O$ & $N \tilde{A} O$ & $N \tilde{A} O$ & $N \tilde{A} O$ & $A$ & $D R^{\prime} S$ \\
\hline 187 & $S I M$ & $N \tilde{A O}$ & $N \tilde{O} O$ & HEDGING & $N \tilde{O} O$ & $A$ & $D R ' S$ \\
\hline 188 & SIM & $N \tilde{A} O$ & $N \tilde{O} O$ & HEDGING & $N \tilde{O} O$ & $A$ & $D R^{\prime} S$ \\
\hline 189 & SIM & $N \tilde{A} O$ & $N \tilde{A} O$ & $N \tilde{O} O$ & $N \tilde{O} O$ & $D$ & $D R ' S$ \\
\hline 190 & SIM & $N \tilde{A} O$ & $N \tilde{A O}$ & HEDGING & $N \tilde{A} O$ & $C$ & $D R^{\prime} S$ \\
\hline 191 & SIM & $N \tilde{O} O$ & $N \tilde{O} O$ & $N \tilde{O} O$ & $N \tilde{O} O$ & $A$ & $D R ' S$ \\
\hline 192 & SIM & $N \tilde{A} O$ & $N \tilde{A} O$ & HEDGING & $N \tilde{A O}$ & $C$ & $D R^{\prime} S$ \\
\hline 193 & SIM & $N \tilde{A O}$ & $N \tilde{A} O$ & HEDGING & $N \tilde{O} O$ & $E$ & IBOVESPA \\
\hline 194 & $N \tilde{A} O$ & $N \tilde{A} O$ & $N \tilde{A} O$ & $N \tilde{O} O$ & $N \tilde{A O}$ & Outras & $D R^{\prime} S$ \\
\hline 195 & $S I M$ & $N \tilde{A} O$ & $N \tilde{A O}$ & HEDGING & $N \tilde{A O}$ & $A$ & $D R ' S$ \\
\hline 196 & SIM & $N \tilde{A} O$ & $N \tilde{A} O$ & $N \tilde{O} O$ & $N \tilde{O} O$ & $B$ & $D R ' S$ \\
\hline 197 & $S I M$ & $N \tilde{O} O$ & $N \tilde{A O}$ & HEDGING & $N \tilde{A O}$ & $D$ & $D R ' S$ \\
\hline 198 & SIM & $N \tilde{A} O$ & $N \tilde{A} O$ & $N \tilde{A} O$ & $N \tilde{A O}$ & $D$ & $D R ' S$ \\
\hline 199 & SIM & $N \tilde{O} O$ & $N \tilde{A} O$ & $N \tilde{O} O$ & $N \tilde{A O}$ & $C$ & $D R^{\prime} S$ \\
\hline 200 & $S I M$ & $N \tilde{A} O$ & $N \tilde{A} O$ & HEDGING & $N \tilde{A} O$ & $B$ & $D R ' S$ \\
\hline 201 & SIM & $N \tilde{O} O$ & $N \tilde{O} O$ & HEDGING & $N \tilde{O} O$ & $A$ & $D R ' S$ \\
\hline 202 & $S I M$ & $N \tilde{A} O$ & $N \tilde{A} O$ & HEDGING & $N \tilde{A} O$ & $D$ & $D R ' S$ \\
\hline 203 & SIM & $N \tilde{A} O$ & $N \tilde{A} O$ & $N \tilde{A} O$ & $N \tilde{A} O$ & $E$ & $D R ' S$ \\
\hline 204 & $S I M$ & $N \tilde{A} O$ & $N \tilde{A} O$ & HEDGING & $N \tilde{A} O$ & $A$ & $D R ' S$ \\
\hline 205 & SIM & SIM & $N \tilde{A} O$ & HEDGING & SIM & $D$ & $D R^{\prime} S$ \\
\hline
\end{tabular}




\section{APÊNDICE}

PLANILHA DE TRABALHO

\begin{tabular}{|c|c|c|c|c|c|c|c|}
\hline & Tem & \multicolumn{2}{|c|}{ Divulgação de Risco } & Atuação no & Divulga & Auditor & Tipo de \\
\hline Cia. & Inst. Fin.? & qualitativo & quantitativo & mercado & fair value & Indep. & instituição \\
\hline 206 & SIM & $N \tilde{O} O$ & $N \tilde{A} O$ & HEDGING & $N \tilde{O} O$ & $F$ & IBOVESPA \\
\hline 207 & SIM & $N \tilde{A} O$ & $N \tilde{A O}$ & HEDGING & $N \tilde{A} O$ & $B$ & $D R ' S$ \\
\hline 208 & SIM & $N \tilde{O} O$ & $N \tilde{A} O$ & HEDGING & $N \tilde{A} O$ & $D$ & IBOVESPA \\
\hline 209 & SIM & $N \tilde{A} O$ & $N \tilde{A O}$ & HEDGING & $N \tilde{A} O$ & $D$ & $D R ' S$ \\
\hline 210 & $S I M$ & $N \tilde{A} O$ & $N \tilde{A} O$ & HEDGING & $N \tilde{A} O$ & $D$ & $D R ' S$ \\
\hline 211 & SIM & $N \tilde{A O}$ & $N \tilde{A} O$ & HEDGING & $N \tilde{A O}$ & $A$ & $D R ' S$ \\
\hline 212 & $S I M$ & $N \tilde{O} O$ & $N \tilde{A} O$ & HEDGING & $S I M$ & $A$ & BANCÁRIA \\
\hline 213 & SIM & $N \tilde{O} O$ & $N \tilde{O} O$ & HEDGING & SIM & $A$ & BANCÁRIA \\
\hline 214 & SIM & $N \tilde{O} O$ & $N \tilde{A} O$ & HEDGING & $N \tilde{O} O$ & $A$ & $D R^{\prime} S$ \\
\hline 215 & SIM & SIM & $N \tilde{O} O$ & HEDGING & $N \tilde{O} O$ & $B$ & $D R ' S$ \\
\hline
\end{tabular}

\section{ANEXO}

\section{OFÍCIO CIRCULAR}

CVM/SEP/SNC/№ 01/2002, ITEM 3

O negócio da companhia compreende a extração e a venda de minério de ferro, para os mercados interno e externo. Os principais fatores de risco de mercado que afetam o negócio da companhia podem ser assim enumerados:

- Risco de crédito: possibilidade de a companhia vir a incorrer em perdas por conta de problemas financeiros com seus clientes, que obriguem-nos a não honrar seus compromissos com a companhia. Para reduzir esse tipo de risco, a companhia celebra com seus clientes contratos acessórios que os obrigam a manter certo nível de endividamento e liquidez. Uma vez ultrapassados esses níveis, há a liquidação antecipada da dívida por parte da companhia e, sendo o caso, a execução de eventuais garantias oferecidas.

- Risco de taxa de câmbio: possibilidade de a companhia vir a incorrer em perdas por conta de flutuações nas taxas de câmbio, que reduzam valores nominais faturado ou aumentem valores captados no mercado. Para reduzir esse tipo de risco, a companhia celebra con- tratos derivativos em mercados de bolsa, conforme demonstrado em quadro específico.

- Risco de preço de minério de ferro: possibilidade de a companhia vir a incorrer em perdas por conta de flutuações de preços de minério de ferro, nos mercados interno e externo. Para reduzir esse tipo de risco, a companhia celebra contratos derivativos em mercados de bolsa e balcão, conforme demonstrado em quadro específico.

\section{Risco de Crédito}

A política de vendas da companhia está intimamente associada ao nível de risco de crédito a que está disposta a se sujeitar no curso de seus negócios. A seletividade de seus clientes, assim como a diversificação de sua carteira de recebíveis, acompanhamento dos prazos de financiamento de vendas por segmento de negócios e limites de posição, são procedimentos que a companhia adota de modo a minimizar eventuais problemas de "default" de seus parceiros comerciais. As mesmas políticas de gestão de risco de crédito são observadas na contratação de derivativos de balcão. A tabela a seguir indica a avaliação dos saldos de contas a receber classificados pelo rating de crédito dos clientes: 


\begin{tabular}{|l|c|c|}
\hline & \multicolumn{2}{|c|}{ Rating de Crédito das Clientes $\left(^{*}\right)$} \\
\hline Prazo dos Contratos & Classificação Moddy's & Classificação Standard \& Poor's \\
\hline Entre 1 e 4 anos & Aa3 & AA- \\
\hline Mais de 4 anos & Aaa & AAA \\
\hline & & \\
\hline Exposição & & AA- \\
\hline Até $R \$ X X X$ milhões & Aa3 & AAA \\
\hline Até $R \$ X X X X$ milhões & Aaa & \\
\hline
\end{tabular}

\begin{tabular}{|l|c|c|}
\hline Diversificação & $31.12 .20 \times 0$ & $31.12 .20 \times 1$ \\
\hline XYZ \& Co. & $6 \%$ & $6 \%$ \\
\hline ABC \& Co. & $8 \%$ & $7 \%$ \\
\hline Wk \& Co. & $7 \%$ & $6 \%$ \\
\hline Outros Clientes $\left(^{*}\right)$ & $79 \%$ & $81 \%$ \\
\hline Total Carteira & $100 \%$ & $100 \%$ \\
\hline
\end{tabular}

$\left({ }^{*}\right)$ demais clientes com participação de no máximo $5 \%$ da carteira de recebíveis

Observação: O exemplo apresentado informa sobre o risco envolvido no financiamento de vendas nos aspectos de qualidade do crédito, a concentração por principais clientes e o prazo de financiamento de vendas. As classificações Moddy's e Standard \& Poor's são exemplos de ratings elaborados por entidades independentes com base em critérios qualitativos e quantitativos. A companhia aberta deve divulgar os critérios escolhidos para a elaboração do rating de seus clientes, esmiuçando o algoritmo utilizado na classificação. Recomenda-se que sejam eleitos critérios objetivos.

\section{Risco de Taxa de Câmbio}

O endividamento e o resultado das operações da companhia são afetados significativamente pelo fator de risco de mercado taxa de câmbio (dólar norteamericano). Foram captados recursos em moeda estrangeira (dólar norte-americano), em 01.01.20X1, para financiar o seu capital de giro.

Em 01.04.20X1, foram obtidos recursos junto a instituição bancária norte-americana (Bank XYZ) para financiar a ampliação da capacidade instalada da companhia, visando a atender os futuros objetivos da companhia de aumento de sua participação no mercado internacional.

A estratégia definida pela companhia, no início do exercício social de 2001, focou preponderantemente o mercado internacional. Com isso, tinha-se a expectativa de o faturamento em dólar da companhia atuar como um hedge natural de seu empréstimo para capital de giro (haveria o balanceamento do seu fluxo de caixa), o que efetivamente aconteceu durante o exercício.

A exposição líquida da companhia, no momento, ao fator de risco de mercado taxa de câmbio é a seguir demonstrado: 


\begin{tabular}{|l|r|r|r|r|}
\hline & \multicolumn{2}{|c|}{ Valor Contábil } & \multicolumn{2}{c|}{ Valor Justo } \\
\hline & $\mathbf{3 1 . 1 2 . 2 0 X 0}$ & $\mathbf{3 1 . 1 2 . 2 0 X 1}$ & $\mathbf{3 1 . 1 2 . 2 0 X 0}$ & $\mathbf{3 1 . 1 2 . 2 0 X 1}$ \\
\hline Empréstimo & $\mathrm{R} \$ \mathrm{XXXXX}$ & $\mathrm{R} \$ \mathrm{XXXXX}$ & $\mathrm{R} \$ \mathrm{XXXX}$ & $\mathrm{R} \$ \mathrm{XXXXX}$ \\
\hline Financiamento & $\mathrm{R} \$ \mathrm{XXXX}$ & $\mathrm{R} \$ \mathrm{XXXX}$ & $\mathrm{R} \$ \mathrm{XXXX}$ & $\mathrm{R} \$ \mathrm{XXXX}$ \\
\hline Total & $\mathrm{R} \$ \mathrm{XXXXX}$ & $\mathrm{R} \$ \mathrm{XXXXX}$ & $\mathrm{R} \$ \mathrm{XXXX}$ & $\mathrm{R} \$ \mathrm{XXXXX}$ \\
\hline & & & & \\
\hline Ativos em dólar & $\mathrm{R} \$ \mathrm{XXX}$ & $\mathrm{R} \$ \mathrm{XXX}$ & $\mathrm{R} \$ \mathrm{XXX}$ & $\mathrm{R} \$ \mathrm{XXX}$ \\
\hline Instrumentos de hedge & $\mathrm{R} \$ \mathrm{XXX}$ & $\mathrm{R} \$ \mathrm{XXX}$ & $\mathrm{R} \$ \mathrm{XXX}$ & $\mathrm{R} \$ \mathrm{XXX}$ \\
\hline Exposição líquida & $\mathrm{R} \$ \mathrm{XXX}$ & $\mathrm{R} \$ \mathrm{XXX}$ & $\mathrm{R} \$ \mathrm{XXX}$ & $\mathrm{R} \$ \mathrm{XXX}$ \\
\hline
\end{tabular}

O método de mensuração utilizado para cômputo do valor justo de todos os elementos, com exceção dos instrumentos de hedge, foi o do fluxo de caixa descontado, considerando expectativas de liquidação ou realização de passivos e ativos e taxas de mercado vigentes na data de corte das informações. Para os instrumentos de hedge, foi utilizada a cotação de mercado dos contratos na data de corte das informações.

\begin{tabular}{|c|c|c|c|c|c|c|}
\hline \multicolumn{7}{|c|}{$\begin{array}{l}\text { Fator de Risco de Mercado: Taxa de câmbio } \\
\begin{array}{l|l}\text { Plano de Amortização/Expectativa de Realização }\end{array}\end{array}$} \\
\hline & $20 \times 2$ & $20 \times 3$ & $20 \times 4$ & $20 \times 5$ & $20 \times 6$ & Total \\
\hline \multicolumn{7}{|l|}{ Moeda US\$: } \\
\hline \multicolumn{7}{|l|}{ 1. Passivo Taxa Flutuante } \\
\hline \multicolumn{7}{|l|}{ Empréstimo Capital de Giro } \\
\hline Principal & $\mathrm{R} \$ \mathrm{XXX}$ & $\mathrm{R} \$ \mathrm{XXX}$ & & & & $R \$ X X X X X$ \\
\hline Juros & $\mathrm{R} \$ \mathrm{XX}$ & $\mathrm{R} \$ \mathrm{XX}$ & & & & $\mathrm{R} \$ \mathrm{XXX}$ \\
\hline Libor Esperada & $X . X \%$ & $\mathrm{X} . \mathrm{X} \%$ & & & & $X . X \%$ \\
\hline \multicolumn{7}{|l|}{ 2. Passivo Taxa Fixa } \\
\hline \multicolumn{7}{|l|}{ Financiamento } \\
\hline Principal & $\mathrm{R} \$ \mathrm{XXX}$ & $\mathrm{R} \$ \mathrm{XXX}$ & $\mathrm{R} \$ \mathrm{XXX}$ & $\mathrm{R} \$ \mathrm{XXX}$ & $\mathrm{R} \$ \mathrm{XXX}$ & $R \$ X X X X X$ \\
\hline Juros & $\mathrm{R} \$ \mathrm{XX}$ & $\mathrm{R} \$ \mathrm{XX}$ & $\mathrm{R} \$ \mathrm{XX}$ & $\mathrm{R} \$ \mathrm{XX}$ & $\mathrm{R} \$ \mathrm{XX}$ & $\mathrm{R} \$ \mathrm{XXX}$ \\
\hline Taxa de Juros Contratada & $X . X \%$ & $\mathrm{X} . \mathrm{X} \%$ & $X . X \%$ & $X . X \%$ & $X . X \%$ & $X . X \%$ \\
\hline 3. Ativo Contas a Receber & $\mathrm{R} \$ \mathrm{XXX}$ & $\mathrm{R} \$ \mathrm{XXX}$ & & & & $R \$ X X X X X$ \\
\hline
\end{tabular}

\begin{tabular}{|c|c|c|c|c|c|}
\hline \multicolumn{6}{|l|}{ Instrumentos de Hedge: } \\
\hline Mercado de Bolsa & Vencimento & Vencimento & Vencimento & Vencimento & Vencimento \\
\hline Contratos Futuros Comprados & $20 \times 2$ & $20 \times 3$ & $20 X 4$ & $20 \times 5$ & $20 \times 6$ \\
\hline a) Valor Nocional & US\$ XXX mil & US\$ XXXX mil & US\$ XX mil & US\$ XX mil & US\$ XX mil \\
\hline b) Taxa contratada a futuro & $\mathrm{R} \$ \mathrm{X}, \mathrm{XX}$ & $\mathrm{R} \$ \mathrm{X}, \mathrm{XX}$ & $\mathrm{R} \$ \mathrm{X}, \mathrm{XX}$ & $\mathrm{R} \$ \mathrm{X}, \mathrm{XX}$ & $\mathrm{R} \$ \mathrm{X}, \mathrm{XX}$ \\
\hline Total $(a \times b)$ & $\mathrm{R} \$ \mathrm{XXXXX}$ & $\mathrm{R} \$ \mathrm{XXXXX}$ & $\mathrm{R} \$ \mathrm{XXXXX}$ & $\mathrm{R} \$ \mathrm{XXXXX}$ & $R \$ X X X X X$ \\
\hline
\end{tabular}

Os contratos derivativos tiveram seus valores nocionais registrados em contas de compensação e os ganhos e perdas, quando realizados financeiramente, registrados em contas de resultado. 


\section{Risco de Preço de Minério de Ferro}

A estratégia adotada pela companhia para minimizar sua exposição ao fator de risco de preço de minério de ferro é a celebração de contratos de liquidação futura (balcão e bolsa), que garantam a realização de seus estoques em um determinado patamar. Seus estoques giram em média três meses, razão pela qual a cada três meses há a rolagem de contratos de liquidação futura.

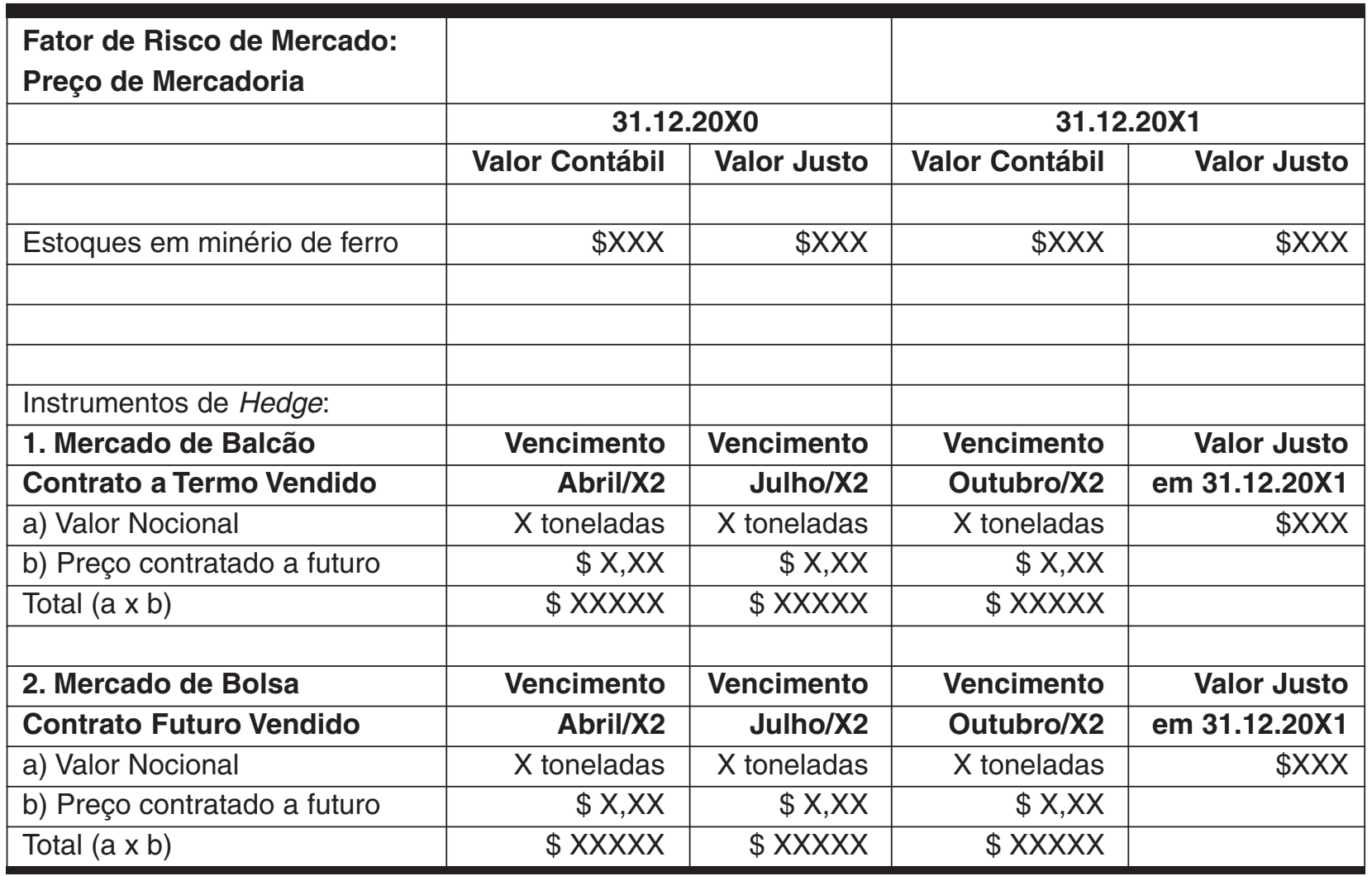

O método de mensuração utilizado para cômputo do valor justo dos estoques e instrumentos de bolsa foi o valor de mercado destes na data de corte das informações. Para os instrumentos de balcão, o método utilizado para cômputo do valor justo foi o do fluxo de caixa descontado, considerando expectativas de liquidação ou realização e taxas de mercado vigentes na data de corte das informações.

Os contratos derivativos tiveram seus valores nocionais registrados em contas de compensação e os ganhos e perdas, quando realizados financeiramente, registrados em contas de resultado. 